\title{
Study The Role of Selenium in The P-Selectin and E-Cadherin Serum Levels in Women with Preeclampsia
}

\author{
Aysel A. Ahmed ${ }^{1}$, Raid J. Al-Timimi, ${ }^{2}$ Enas Adnan Abdulrasol ${ }^{\mathbf{3}}$ \\ ${ }^{1}$ Department of Chemistry and Biochemistry, Collage of Medicine, Al-Nahrin university. Baghdad Iraq
}

\begin{abstract}
Preeclampsia (PE) is characterized by endothelial dysfunction with vasoconstriction due to cell adhesion molecules or mediators released by defective placentation. Platelet selectin (P-selectin) and epithelial cadherin (E-cadherin) are the cell adhesion molecules, is elevated in many inflammatory conditions including PE.
\end{abstract}

Objective: To investigate if P-selectin and E-cadherin levels can be used as a marker for adverse outcomes in pregnancy complicated with PE.

Patients and Method: This study involved 90 pregnant women in their third trimester of pregnancy divided into2 group:

Group A: involves 45 pregnant women with PE.

Group B: involve 45 healthy pregnant women and normal fetal growth (Control group).

Result: The results show there were a high significant increase in the mean values of P-selectin with high increase in the mean values of E-cadherin in PE group when compared to control group.

ROC curve shows sensitivity and specificity and false positive rate for predication of PE by P-selectin and E-cadherin in control and patients groups, and the maximum accuracy for the P-selectin and E-cadherin in discrimination between control and PE patients groups.

Conclusions: There is an inverse relationship between cell adhesion molecules and selenium in PE compared with normal pregnancy.

High level of serum P-selectin and E-cadherin are associated with the presence PE, which provide additional information for predicting of $\mathrm{PE}$ and preventing its complications and for better understanding pathogenesis of PE.

Key words: platelet-selectin and epithelial-cadherin, with preeclampsia \& with normal pregnancy.

\section{Introduction}

Preeclampsia (PE) is a disorder of pregnancy, is a multisystem syndrome that is primarily, characterized by the onset of high blood pressure (HBP) (hypertension) and often a significant amount of protein in the urine (proteinuria). When it arises, the condition can appear any time from the twenty weeks of pregnancy ${ }^{(1)}$. The exact causes of PE and eclampsia - a result of a placenta that doesn't function properly, insufficient blood flow to the uterus could be associated. Genetics plays a role as well, and have many risk factor for PE is most often seen in first-time pregnancies, in pregnant teens, and in women over 40 years old While it is defined as occurring in women have never had high blood pressure before, other risk factors include:

A history of high blood pressure prior to pregnancy.

A history of preeclampsia.

Having a mother or sister who had preeclampsia.

A history of obesity. 
History of diabetes, kidney disease , lupus , in the pathogenesis of several pathologies including PE. or rheumatoid arthritis.

P-selectin functions as a cell adhesion molecule (CAM) on the surfaces of activated endothelial cells, which line the inner surface of blood vessels, and activated platelets. In inactivated endothelial cells, it is stored in granules called WeibelPalade bodies. In inactivated platelets P-selectin is stored in $\alpha$-granules of platelets, have been shown to correlate with the level of oxidative stress in pregnancy ${ }^{(2)}$.

Other names for P-selectin include CD62P, Granule Membrane Protein 140 (GMP-140) and Platelet Activation-Dependent Granule to External Membrane Protein (PADGEM) ${ }^{(3)}$.

Cadherin (named for "calcium-dependent adhesion") are a type of cell adhesion molecule (CAM) that is important in the formation of adherence junctions to bind cells with each other ${ }^{(4)}$. E- Cadherin is a class of type- 1 transmembrane proteins. It is dependent on calcium $\left(\mathrm{Ca}^{2+}\right)$ ions to function.

Cell-to-cell adhesion mediated by E- Cadherin plays an important role in tissue differentiation, holding the tissues together as they form during embryonic development.

\section{Role of Adhesion in Preeclampsia:}

Increase expression of adhesion molecule interfere with homeostasis. For example adhesion molecules can activate platelets through their contact with exposed epithelial wall ${ }^{(5 ; 6)}$. Abnormal active platelet are involved

\section{Patients and Methods}

A case control study was conducted on 90 pregnant women (aged 18-45 years old) from the obstetrics and gynecology department of Al- Immamain Al-Kadhmain Hospital and Baghdad Teaching Hospital, after taking an approval from institutional review board (I.R.B.) of the Medical Collage / Al-Nahrain university and taking a written informed consent from all women in the study.

The subject in the study were 45 pregnant women with PE patient and 45 health pregnant women with gestational age matching to the cases at sampling, which remain normotensive and deliver a healthy baby. Blood pressure for each pregnant women was measured twice in four-hour intervals while resting, at third trimester as well as protenuria detected in urine sample by a simple dip-stick test.

The blood sample were taken from each women that participate in the study and used to measure P-selectin and E-cadherin by using enzyme linked immunosorbent assay (ELISA) method.

\section{Result}

Serum Levels of Adhesive Molecules

Mean serum level of P-selectin in PE patients was $261.69 \pm 101.64 \mathrm{ng} / \mathrm{mL}$ which was higher than that of controls $(204.13 \pm 85.68 \mathrm{ng} / \mathrm{mL})$ with a significant difference (Figure 1).

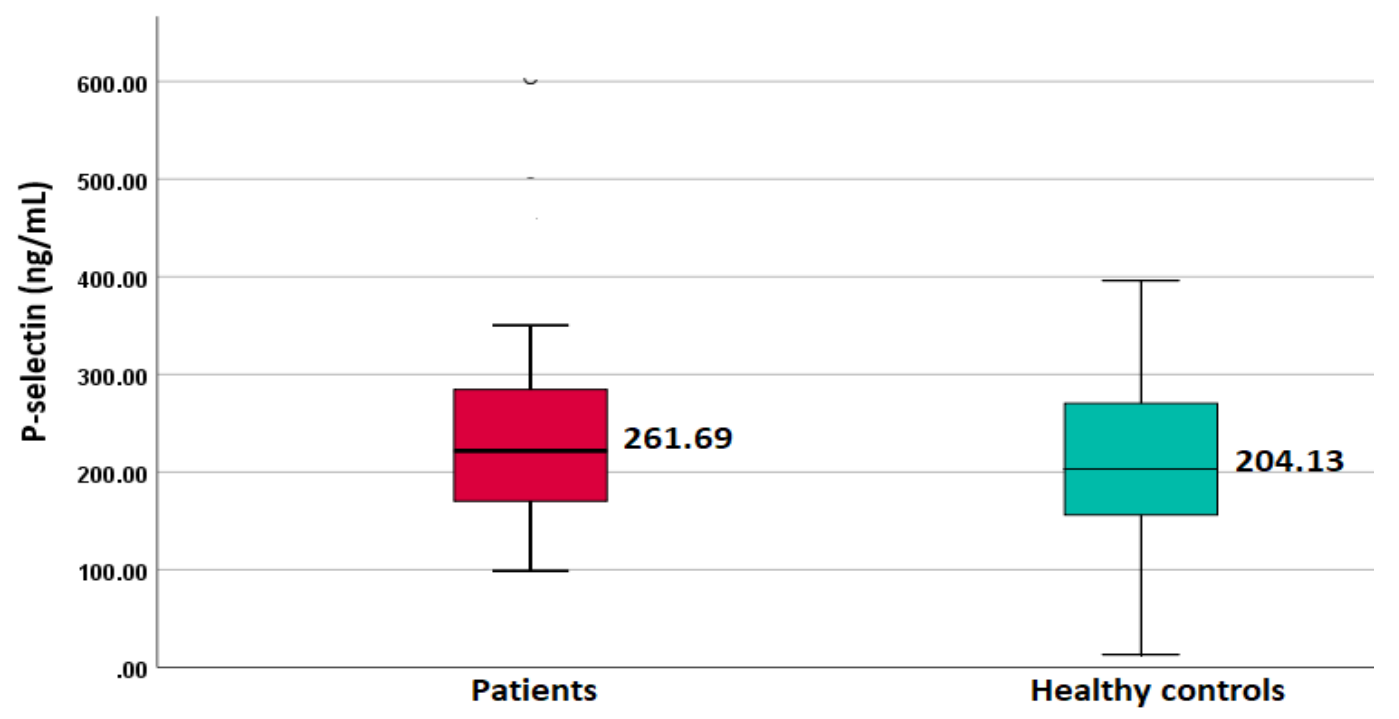

Figure 1: Mean serum level of $\mathrm{P}$-selecting in $\mathrm{PE}$ patients and healthy controls 
Similarly, mean serum level of E-cadherin in patients $(69.45 \pm 15.26 \mathrm{ng} / \mathrm{mL})$ was higher than that of controls $(58.6 \pm 21.69 \mathrm{ng} / \mathrm{mL})$ with a significant difference (Figure 2).

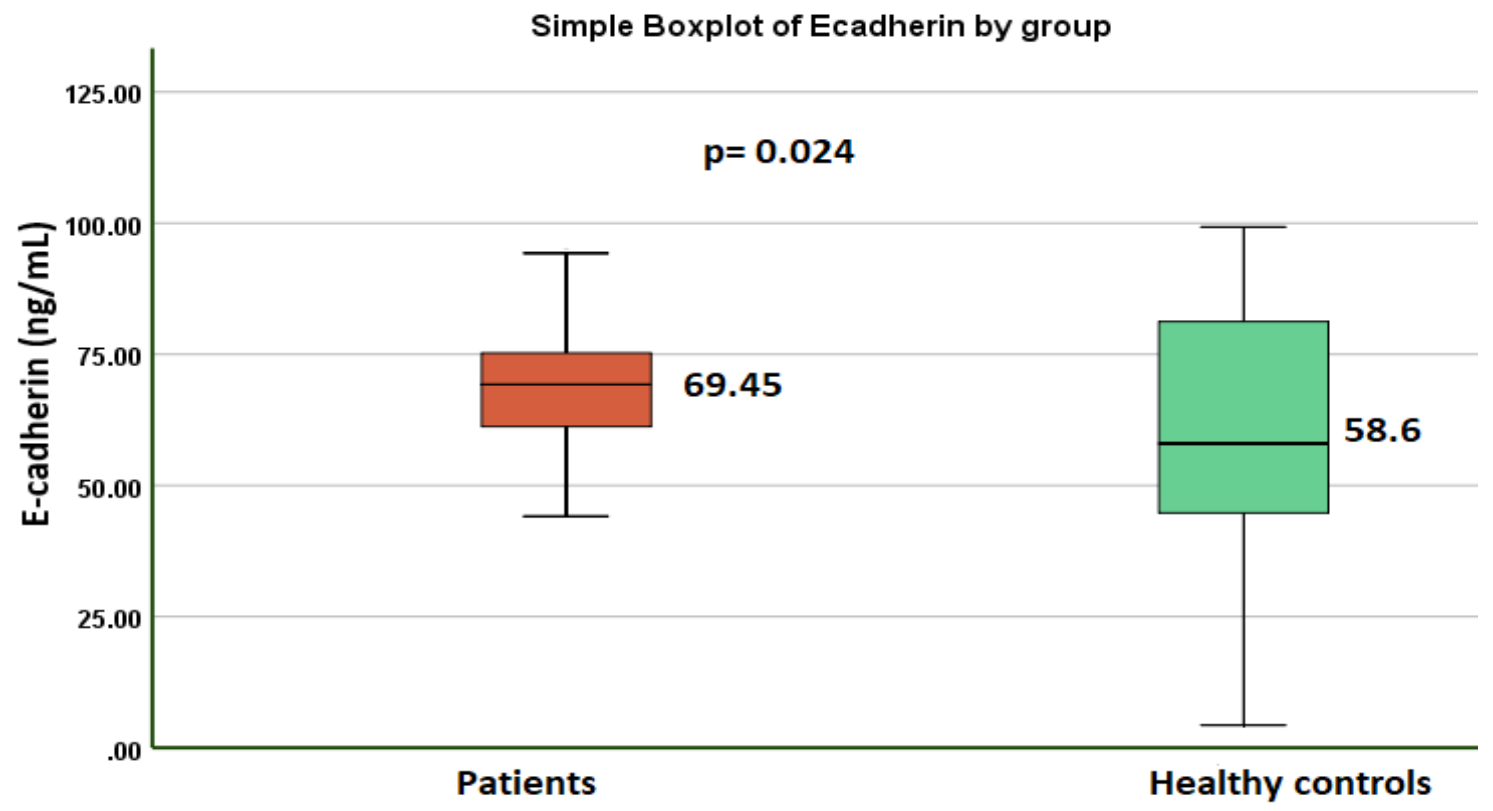

Figure 2: Mean serum level of E-cadherin in PE patients and healthy controls

\section{Diagnostic Value of Adhesive Molecules}

Receiver operating characteristic curve was used to explore the diagnostic value of adhesive molecule in the context of discrimination between PE patients and healthy controls (Figure 3).

For P-selectin, the AUC was $0.586,95 \% \mathrm{CI}=0.469$ -
$0.704, p=0.159$. The sensitivity and specificity of the test atcut off values of P-selectin $=211.0 \mathrm{ng} / \mathrm{mL}$ were 0.60 and 0.556 respectively.

For E-cadherin, the AUC was $0.617,95 \% \mathrm{CI}=$ $0.495-0.739, p=0.056$. The sensitivity and specificity of the test atcut off values of E-cadherin $=64.87 \mathrm{ng} / \mathrm{mL}$ were 0.622 for both.

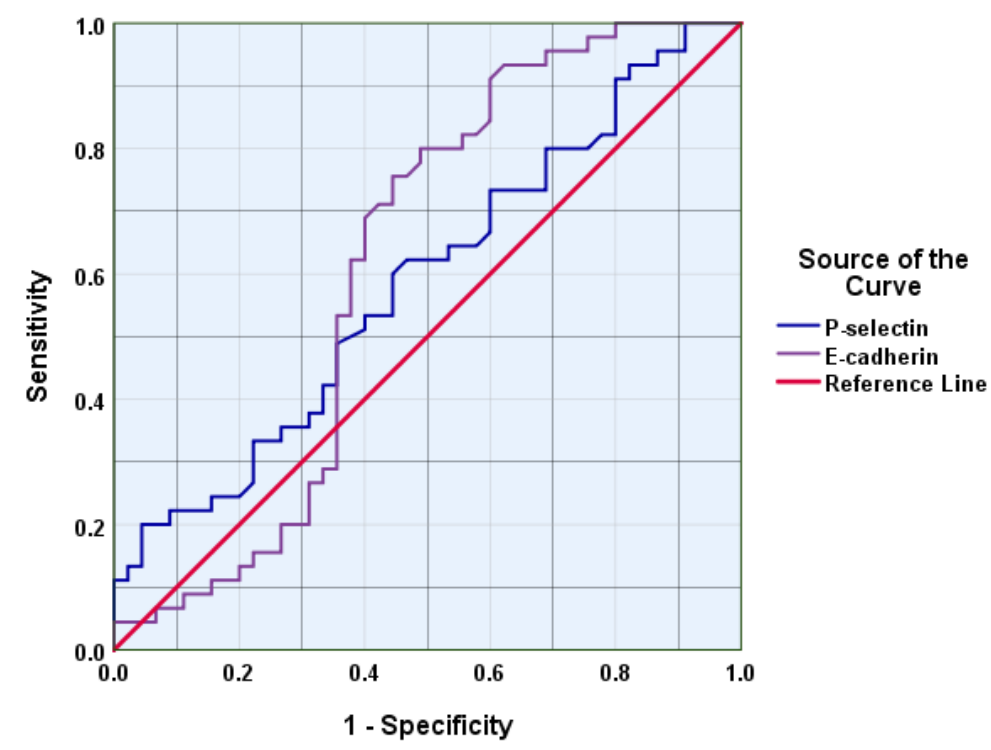

Figure 3: Receiver operating curve for P-selectin and E-cadherin in the context of discrimination between PE patients and healthy controls 
The combination of the two markers did not improve the discriminative value (Figure 4). The AUC was 0.613, $95 \% \mathrm{CI}=0.496-0.729, \mathrm{p}=0.056$. The sensitivity and specificity of the test at the corresponding cut off values were 0.60 and 0.51 respectively.

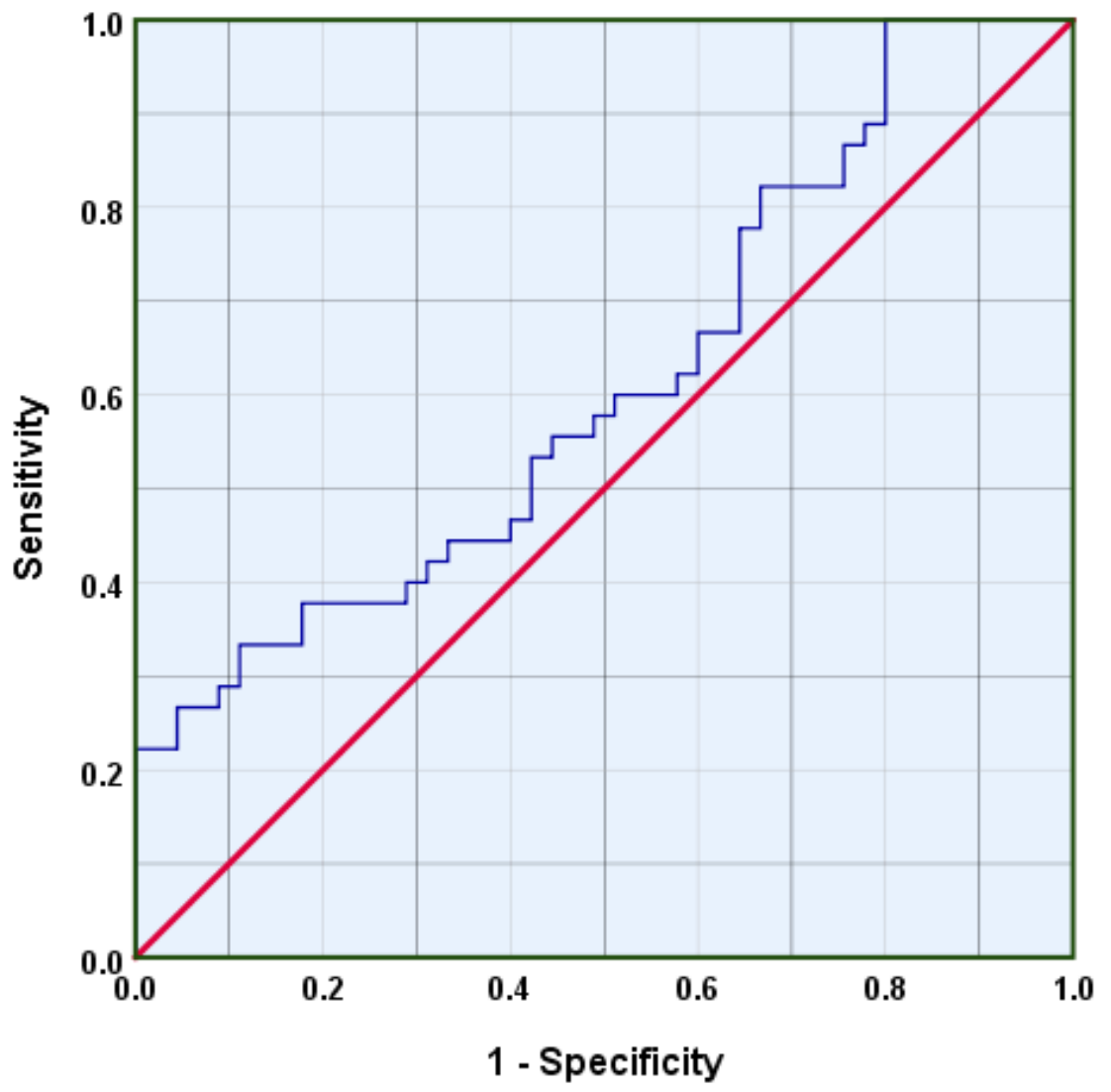

Figure 4: Receiver operating curve for a combination of P-selectin and E-cadherin in the context of discrimination between PE patients and healthy controls

\section{Discussion}

In the present study, markers were chosen to reflect inflammation, endothelial dysfunction and poor placentation which are the hallmark of PE. Previous studies which have done on the these markers in other similar clinical situations such as CVD, had shown great association of the markers with the disease processes.

Nevertheless, these clinical condition are known to share more than one physiological and vascular factors. So that this study aimed to investigate the role of current markers with PE development.

\section{Serum Levels of Adhesive Molecules}

In the current work, the mean level of P-selectin was found to be significantly higher in women with PE compared with the control group. Presented a significantly higher expression of P-selectin in PE than in apparently healthy pregnant women.
Same results of increased levels of sP-selectin in preeclamptic pregnancies were obtained by ${ }^{(7)}$. The authors proposed that their findings support an inflammatory model for PE, while the endothelial cell activation might be a secondary to a primary inflammatory response. However, ${ }^{(8)}$ observed significantly increased levels of both P-selectin and sP- selectin form in women with $\mathrm{PE}$ in comparison with normal pregnant women and came to the result that normal pregnancy is connected with increased platelet activation followed by further alteration in these parameters in pregnancies complicated by hypertension.

Also, the present findings are in accordance with the results reported by ${ }^{(9)}$, who suggested that the elevated level of the P-selectin is associated with PE (Figure 1), $\mathrm{sP}$-selectin acts as a marker for platelet and endothelial activation and as an initiator of the pro-coagulant state as well as playing an important role in atherosclerosis. The current results of elevated $\mathrm{SP}$-selectin in $\mathrm{PE}$ seem 
to confirm that these processes occur in preeclamptic pregnant women and the excess circulating sP-selectin aid to the pathogenesis of this pregnancy-specific disorder.

There are two sources of sP-selectin. A minor proportion of this marker is produced through alternative splicing of mRNA which remover the exon that coding the transmembraneous domain.

However, the main proportion of sP-selectin is primarily derived from proteolytic cleavage of the ectoderm part of membranous P-selectin.

Interestingly, shedding of sP-selsectin requires leukocyte adhesion to activate platelets or endothelial cells through interaction of PSGL-1 on leukocyte ${ }^{(10)}$.

$\mathrm{PE}$ involves an endothelial cell activation to coagulation hyperactivity and inflammation, which implies the availability of PSGL-1 for activation of platelets and endothelial cells with eventual increase in sP-selectin shedding.

The circulating platelet count is reduced reflecting a decreased platelet lifespan ${ }^{(11)}$. Features of platelet activation in $\mathrm{PE}$ include an increased expression of platelet surface P-selectin (CD62P) ${ }^{(6)}$. As can be observed from receiver operating characteristic curve (ROC) (Figure 3). Analyses of P-selectin demonstrated the low descrimitive value of this marker to diagnose PE both in terms of sensitivity and specificity. The sensitivity was 0.60 and specificity 0.556 respectively. And the AUC was $0.586,95 \% \mathrm{CI}=0.469-0.704, \mathrm{P}=0.159$. This is because $\mathrm{sP}$-selectin can elevated in many pathologies. For example in a case-control study, including women with polycystic ovary syndrome (PCOs) and healthy control women, found that $\mathrm{sP}$-selectin levels were significantly higher in PCOs women than controls.

The current study revealed a significant elevation in serum level of E-cadherin in PE compared with control. This result in accordance with during human placental development, cytotrphoblast(CTB) differentiate and fuse with the syncytio-trophoblast cell layer (STB), allowing for growth of the syncytium and for re-epithelization of aged or damaged regions of villi. This process of trophoblast turnover is regulated to ensure normal fetal development. Localization and expression of cadherins are highly relevant to the morphology of trophoblasts at different stages of placental development. E-cadherin, a classical cell-cell adhesion molecule, is expressed in villous $\mathrm{CTB}$, but is lost when they differentiate and syncytialise, resulting in the absence of E-cadherin expression in the STB of both healthy 1 and 2 trimester placentae ${ }^{(12)}$. The reduction of E-cadherin expression from 1 trimester to 3 trimester is reported in normal term placenta ${ }^{(13)}$. A number of studies have shown that the expression of E-cadherin was increased in PE resulting in CTB proliferation ${ }^{(14)}$, although more recent studies have shown the reduced expression of E-cadherin in $\mathrm{PE}$, or no changes in E-cadherin expression in TNF- $\alpha$ induced reduction of trophoblastinvasion ${ }^{(15)}$. Elevated expression of E-cadherin raise the proliferation of CTB and inhibits their differentiation into STB in PE, which suggests the alterations of E-cadherin levels are potentially important to the pathogenesis of PE ${ }^{(16)}$. Consistent with other studies, this data showed that E-cadherin was expressed in a higher level in the preeclamptic patient women compared with normotensive pregnant women. Studies have shown the villous trophoblast undergoes increased apoptosis in preeclampsia and the increased apoptosis was observed in STB ${ }^{(17)}$.

As can be observed from receiver operating characteristic curve (ROC) (Figure 3), analyses of E-cadherin demonstrated poor diagnostic value of this marker to diagnose PE. The sensitivity and specificity was 0.622 for both.

The combination of the two markers did not improve the discriminative value (Figure 4). The AUC was 0.613, $95 \% \mathrm{CI}=0.496-0.729, \mathrm{p}=0.056$. The sensitivity and specificity of the test at the corresponding cut off values were 0.60 and 0.51 respectively.

\section{Conclusions}

There is an inverse relationship between cell adhesion molecules and selenium in PE compared with normal pregnancy.

High level of serum P-selectin and E-cadherin are associated with the presence $P E$, which provide additional information for predicting of PE and preventing its complications and for better understanding pathogenesis of PE.

Ethical Clearance: The Research Ethical Committee at scientific research by ethical approval of both environmental and health and higher education and scientific research ministries in Iraq

Conflict of Interest: The authors declare that they 
have no conflict of interest.

Funding: Self-funding

\section{References}

1. Nakanishi S, Aoki S, Nagashima A, Seki K. Incidence and pregnancy outcomes of superimposed preeclampsia with or without proteinuria among women with chronic hypertension. Pregnancy Hypertension: An International Journal of Women's Cardiovascular Health. 2017;7:39-43.

2. Chamy Pico VM, Lepe J, Catalan A, Retamal D, Escobar JA, Madrid EM. Oxidative stress is closely related to clinical severity of pre-eclampsia.

3. McEver RP, Beckstead JH, Moore KL, MarshallCarlson L, Bainton DF. GMP-140, a platelet alphagranule membrane protein, is also synthesized by vascular endothelial cells and is localized in WeibelPalade bodies. The Journal of clinical investigation. 1989;84(1):92-9.

4. Alimperti S, Andreadis ST. CDH2 and CDH11 act as regulators of stem cell fate decisions. Stem cell research. 2015;14(3):270-82.

5. Abass AE, Abdalla R, Omer I, Ahmed S, Khalid A, Elzein H. Evaluation of platelets count and indices in pre-eclampsia compared to normal pregnancies. IOSR Journal of Dental and Medical Sciences (IOSR-JDMS). 2016;1(15):5-8.

6. Holthe MR, Staff AC, Berge LN, Lyberg T. Different levels of platelet activation in preeclamptic, normotensive pregnant, and nonpregnant women. American journal of obstetrics and gynecology. 2004;190(4):1128-34.

7. Chaiworapongsa $\mathrm{T}$, Romero R, Yoshimatsu J, Espinoza J, Kim YM, Park K, Kalache K, Edwin S, Bujold E, Gomez R. Soluble adhesion molecule profile in normal pregnancy and pre-eclampsia. The journal of maternal-fetal \& neonatal medicine. 2002;12(1):19-27.

8. Karalis I, Nadar SK, Al Yemeni E, Blann $\mathrm{AD}$, Lip GY. Platelet activation in pregnancyinduced hypertension. Thrombosis research. 2005;116(5):377-83.
9. Laskowska M, Laskowska K, Oleszczuk J. Elevated maternal serum sP-selectin levels in preeclamptic pregnancies with and without intrauterine fetal growth restriction, but not in normotensive pregnancies complicated by isolated IUGR. Medical science monitor: international medical journal of experimental and clinical research. 2013;19:118.

10. Paniker CJ, Ghosh S. Paniker's textbook of medical parasitology. JP Medical Ltd; 2017 Sep 14.

11. Haram K, Mortensen JH, Wollen AL. Preterm delivery: an overview. Acta obstetricia et gynecologica Scandinavica. 2003;82(8):687-704.

12. Fisher SJ, Cui TY, Zhang L, Hartman L, Grahl K, Zhang GY, Tarpey J, Damsky CH. Adhesive and degradative properties of human placental cytotrophoblast cells in vitro. The Journal of cell biology. 1989;109(2):891-902.

13. Floridon C, Nielsen O, Hølund B, Sunde L, Westergaard JG, Thomsen SG, Teisner B. Localization of E-cadherin in villous, extravillous and vascular trophoblasts during intrauterine, ectopic and molar pregnancy. MHR: Basic science of reproductive medicine. 2000;6(10):943-50.

14. Li HW, Cheung AN, Tsao SW, Cheung AL. Expression of e-cadherin and beta-catenin in trophoblastic tissue in normal and pathological pregnancies. International journal of gynecological pathology. 2003;22(1):63-70.

15. Xu B, Nakhla S, Makris A, Hennessy A. TNF- $\alpha$ inhibits trophoblast integration into endothelial cellular networks. Placenta. 2011;32(3):241-6.

16. Brown LM, Lacey HA, Baker PN, Crocker IP. E-cadherin in the assessment of aberrant placental cytotrophoblast turnover in pregnancies complicated by pre-eclampsia. Histochemistry and cell biology. 2005;124(6):499-506.

17. Heazell AE, Taylor NN, Greenwood SL, Baker PN, Crocker IP. Does altered oxygenation or reactive oxygen species alter cell turnover of BeWo choriocarcinoma cells? Reproductive biomedicine online. 2009;18(1):111-9. 